ENSAYO 



\title{
EL RETO DE LAS COMPETENCIAS Y LA ENSEÑANZA UNIVERSITARIA
}

\author{
THE CHALLENGE OF SKILLS AND UNIVERSITY EDUCATION
}

Olga González Sarmiento ${ }^{1}$

\section{RESUMEN}

El presente artículo desarrolla el tema de las competencias en la educación superior universitaria, la variedad de definiciones existentes sobre el constructo, los tipos de competencias por los que se trabaja en la universidad y las implicancias con respecto a la formación profesional en un enfoque basado en competencias de aprendizaje.

\section{Palabras clave}

Habilidades, capacidades, competencias genéricas, competencias específicas, competencias clave, educación superior.

\section{ABSTRACT}

This paper develops the topic of competencies in university education, the variety of existing definitions of the construct, the types of skills for those working at university and the implications concerning professional training, focused on learning competences.

\section{Keywords}

Skills, capacities, generic skills, specific skills, key competences, higher education

Educar es, indudablemente, una de las mejores ocupaciones a la que puede dedicarse un ser humano, sin embargo, algunos consideran que se trata de una tarea fácil que cualquiera, medianamente preparado, puede realizar con éxito.

Esa premisa no es cierta. El acto de educar involucra a toda la persona del educando, no solamente su aspecto cognitivo. Implica estar conscientes de que en cada uno de ellos existe un potencial a ser desarrollado, un potencial que correría el riesgo de desperdiciarse si, durante el proceso de formación, quien educa se circunscribe solamente a desarrollar la temática de un curso.
Es en este contexto que se empieza a hablar sobre competencias en el nivel de Educación Superior. Al respecto, existe un cierto acuerdo entre los autores que profundizan sobre el mencionado constructo. En tal sentido, se entiende por competencia a un conjunto de conocimientos, habilidades y actitudes que un profesional debe mostrar en el ejercicio de una determinada disciplina.

Existen diversas definiciones de lo que se denomina competencia. Para el Proyecto Tuning Europa, por ejemplo, "las competencias representan una combinación dinámica de conocimiento, comprensión, capacidades y habilidades" (Beneitone et ál., 2007)

\footnotetext{
1 La Dra. Olga González Sarmiento se formó en la Universidad Femenina del Sagrado Corazón y en la Pontificia Universidad Católica del Perú, actualmente centra sus intereses en temas asociados a la educación superior y al campo de la psicología clínica.
} 
Por otro lado, también se asocia al término competencia con la idea del "saber hacer", sin embargo, según Le Bortef, más exacto sería asociarla, más bien, al "saber actuar" en determinada situación. Para Guy Le Bortef (2001) se trata de "un saber actuar en un contexto de trabajo". Perrenoud (2000), por su parte, considera a la competencia como la capacidad para movilizar diversos recursos cognitivos para hacer frente a un tipo de situaciones.

Sintetiza, dicho autor, que el haber desarrollado una competencia implica ser capaz de transferir lo aprendido, de tener autonomía en el aprendizaje y de resolver problemas en un determinado contexto.

Por otro lado, la definición proporcionada por F. Weinert (2001), involucra todos los recursos mentales que las personas emplean para realizar las tareas importantes, para adquirir conocimientos y para conseguir un buen desempeño.

La Unión Europea también aporta una definición acerca del término. Según su propuesta, se denomina competencia a la "combinación de destrezas, conocimientos, aptitudes y actitudes, así como la disposición de aprender, además del saber cómo hacerlo".

Cita la siguiente definición de la UE, Juan López (2012) en su libro Las competencias básicas como lugar de encuentro:

Las competencias clave representan un paquete multifuncional y transferible de conocimientos, destrezas y actitudes que todos los individuos necesitan para su realización y desarrollo personal, inclusión y empleo, por lo que estas deberían haber sido desarrolladas para el final de la enseñanza, o formación obligatoria, y deberían actuar como la base para un posterior aprendizaje a lo largo de la vida"p.219

Como es posible observar en base a lo previamente descrito, las definiciones sobre competencia varían y son percibidas desde dos perspectivas totalmente opuestas por los autores. Por un lado, se consideran a partir de una perspectiva "social" del individuo y por otra, desde una perspectiva más "personal" del individuo.

Desde la primera perspectiva, se destaca la capacidad para realizar tareas y desempeñar roles que respondan a determinados estándares esperados; desde la segunda, se destacan los rasgos personales requeridos para la ejecución de un trabajo determinado.

En el contexto de la perspectiva "personal", Le Bortef (2001) afirma que

la persona competente es aquella que es capaz de realizar un conjunto de actividades según ciertos criterios deseables, en un contexto particular, eligiendo y movilizando un equipamiento doble de recursos: recursos personales (conocimientos, saber-hacer, cualidades, cultura, motivaciones, afectividad...) y recursos para manejo de información lequipamiento incorporado para obtenerla: banco de datos, redes documentales, redes de experiencia especializada, etc.) p. 19.

Sin embargo, en el campo de las competencias no solo es posible identificar variedad en la forma cómo se denomina o se percibe qué es una competencia, también se hace una interesante diferenciación acerca de los diferentes tipos de competencias existentes según su grado de generalidad o especificidad.

Así, se habla de competencias básicas, genéricas y específicas. De esta manera, las competencias básicas están asociadas a habilidades y conocimientos básicos que debe poseer toda persona para desenvolverse de manera conveniente. Por ejemplo, las habilidades de comunicación, de escucha, las de cálculo matemático, las de redacción, etc.

Por otro lado, existen las competencias denominadas genéricas. En este tipo de competencias se encuentran todas aquellas que reúnen conocimientos y habilidades que son comunes, en el caso de la educación superior, a cualquier profesional. Estas competencias, 
por tanto, deben ejercitarse en todos los planes de estudio y deben estar presentes, de manera trasversal en la programación de los cursos, pues son de mucha relevancia para desempeñarse de manera exitosa en una profesión. Entre ellas se encuentran por ejemplo, las capacidades de trabajar en equipo, de negociar, de planificar.

El informe Eurydice (2002) se ocupó del estudio de tales competencias, considerando que están relacionadas con una mejor organización del propio aprendizaje, en la manera como el educando se desenvuelve en las relaciones sociales $e$ interpersonales $y$, por supuesto, en cómo se comunica. Estas conclusiones conducen, junto con otros aportes, a la propuesta del cambio general propuesto para la educación, de un enfoque centrado en la enseñanza hacia un enfoque centrado en el aprendizaje.

Del mismo modo, el estudio internacional PISA (2000) enfatizó la importancia de la adquisición, por parte de los estudiantes, de competencias más amplias con miras a alcanzar un aprendizaje exitoso. Por lo que, aparte del rendimiento en lectura y matemáticas, también incluyó la evaluación de competencias genéricas tales como la motivación del alumnado, sus actitudes $y$ habilidades para regular el propio aprendizaje, etc.

Finalmente, en esta clasificación, se encuentran las competencias específicas, que son propias de una determinada profesión. Es decir, se refieren a las capacidades y a los conocimientos relacionados con cada una de las disciplinas académicas y con su desempeño laboral. Son competencias propias de un determinado perfil formativo y pueden ser compartidas por campos afines. Por ejemplo, realizar cirugías, diseñar proyectos arquitectónicos, elaborar un estado de costo-beneficio, resolver operaciones de matemáticas avanzadas, etc.

El Consejo y el Parlamento Europeo adoptaron, a finales de 2006, un marco de referencia que identificara y definiera las competencias clave que necesitan los ciudadanos para su realización personal, inclusión social, ciudadanía activa y empleabilidad.

Esa clasificación de competencias pretendía, de alguna manera, ser una referencia para los responsables de formular políticas educativas, para los educadores $y$, por supuesto, para los empleadores y estudiantes, con el fin de impulsar iniciativas en pos de objetivos comúnmente acordados en pro a mejoras sociales y búsqueda del desarrollo en cada región.

En este contexto, pueden reconocerse ocho ámbitos específicos en los que es posible identificar la necesidad de trabajar desde la educación por el logro de competencias clave. Estas serían las siguientes: comunicación en lengua materna, comunicación en lengua extranjera, competencia en matemática, ciencia y tecnología, competencia digital, competencia para aprender a aprender, competencias interpersonales y cívicas, espíritu emprendedor y expresión cultural.

Sobre el tema de competencias clave, la Comisión Europea (2004) considera que una 'competencia clave' o genérica, según otras denominaciones, es crucial para tres aspectos de la vida:

a. realización y desarrollo de la persona a lo largo de la vida, lo que se puede asociar al capital cultural: dicho tipo de competencias deben permitir a los sujetos de la formación, perseguir objetivos personales conducidos por sus intereses, sus aspiraciones y el deseo de continuar aprendiendo a lo largo de la vida;

b. inclusión y una ciudadanía activa que correspondería al denominado capital social: las competencias clave deberían permitir a todos participar de manera efectiva como ciudadanos activos en la sociedad;

c. aptitud para el empleo, que vendría a entenderse sobre la forma como está preparado en términos de capital humano: la capacidad de todas y cada una de las personas de obtener un puesto de trabajo decente en el mercado laboral. 
Por otro lado, en el ámbito universitario, dichas competencias clave o genéricas son consideradas apropiadas para la mayoría de las carreras y están asociadas a la formación universitaria en sí porque incluyen las habilidades, los conocimientos y las actitudes consideradas de utilidad para cualquier sociedad.

En este contexto, entonces, podemos afirmar que todas las interpretaciones previamente citadas, aluden a un carácter eminentemente práctico de las competencias.

Es decir, para saber si alguien es competente, es indispensable observarlo cuando trabaja, no se puede decir que esa persona es competente solo porque "sabe" cómo debe hacer, sino cuando es observado durante su desempeño y se concluye que realiza la acción requerida de manera correcta. Sin embargo, tampoco puede afirmarse de manera categórica que si una persona es observada durante su desempeño y está desenvolviéndose de manera acertada, la competencia ya está lograda. Afirman los expertos en el tema que es necesario, además, conocer la actitud y la disposición con que ejecuta dicha tarea.

Por tanto, puede afirmarse, en el contexto de la educación superior, que el logro de una competencia involucra tres componentes:

a. Habilidades que se apoyan para realizar con éxito una determinada tarea académica o profesional.

b. Uso de conocimientos generales propios de diversas disciplinas o áreas de estudio $\mathrm{y}$, por supuesto, uso de conocimientos específicos de la propia profesión.

c. Desempeño con la actitud y disposición adecuadas, en concordancia con determinados valores humanos, sociales, culturales, con uso de una correcta autorregulación en el actuar.

Es así como la elección de un determinado enfoque de enseñanza y evaluación a través de competencias debería reflejar un compromiso por parte de la institución que lo adopta, orientado al aprendizaje (y a la evaluación de este) a través de la ejecución de habilidades y la formación en las actitudes correspondientes, que contribuyan a la construcción del profesional requerido por la sociedad a la que pertenece.

Y, ¿qué es lo que requiere una sociedad de sus profesionales?

Pues, ante todo, que un profesional tenga desarrolladas su capacidad de análisis, de síntesis, de resolución de problemas, de crítica y de autocrítica, que sea capaz de generar nuevas ideas y proyectos para sí mismo/a y para su entorno $y$, por supuesto, que cuente con un espíritu emprendedor que lo conduzca al logro de cada vez mayores y mejores metas, en las que estará involucrada, sin duda, su iniciativa personal.

Sin embargo y como sabemos, el aspecto cognitivo de la persona no lo es todo, cada sociedad requiere, además, que sus profesionales se relacionen de manera proactiva, entre sí, con sus compañeros de trabajo, sus autoridades o sus subordinados, que sean capaces de trabajar en equipo, de manera colaborativa y, que sean capaces de reconocer y controlar sus emociones.

Asimismo, una sociedad de aprendizaje permanente requiere que sus profesionales sean activos $y$ eficientes buscadores de información de calidad en línea, que sean capaces de utilizarla, procesarla y luego, generar nuevos conocimientos en favor de su comunidad.

Finalmente, solo el profesional que se encuentra en el nivel de aprendiz experto sabe lo necesario que es saber regular su propio proceso de aprendizaje, monitorearlo y generar nuevos y mejores aprendizajes, construidos en base a lo que ya posee, haciendo uso de sus procesos metacognitivos y, por supuesto, ser capaz de continuar haciéndolo a lo largo de su vida, lo que denominan algunos, que es la mejor manera de ser un aprendiz a lo largo de su vida. 
Para trabajar estos fines desde la universidad, sería necesario interpretar información, es decir hacer uso de la comprensión a través de las habilidades de comparar, clasificar, analizar, sintetizar, secuenciar, averiguar razones $y$ extraer conclusiones.

Aparte de comprender es sumamente necesario estar preparado para evaluar la información que se recibe o a la que se accede, es decir, hacer uso del pensamiento crítico, desarrollando habilidades que le permitan identificar fuentes informativas de calidad, interpretar causas, predecir efectos, razonar.
Desde el pensamiento de tipo creativo, es necesario que la enseñanza le proporcionemos al estudiante la oportunidad de elaborar ideas, establecer relaciones, crear metáforas, etc.

Es así que, desde las estrategias que utilicemos en el aula, pasando por las situaciones de aprendizaje que generemos, hasta la forma en que evaluemos a nuestros estudiantes, todo este conjunto nos va a posibilitar que, efectivamente, formemos en un enfoque de competencias de aprendizaje y, paralelamente, contribuyamos, desde nuestro quehacer diario a la formación de los profesionales que nuestra sociedad requiere. 


\section{REFERENCIAS}

Banco Mundial. (2003): Construir Sociedades de Conocimiento: nuevos desafíos para la Educación Terciaria. Washington: Banco Internacional de Reconstrucción y Fomento / Banco Mundial. Resources/Documents/Constructing-KnowledgeSocieties/CKS-spanish.pdf

Beneitone, P., Esquetini, C., González, J., Maelta, M. M., Siufi, G. y Wagenaar, R. (Eds.). (2007). Informe final proyecto Tuning. 2004-2007. Reflexiones y perspectivas de la educación superior en América Latina. Bilbao: Universidad de Deusto.

Recuperado de: http://tuning.unideusto. org/tuningal/

Comisión Europea. (2004). Competencias clave para un aprendizaje a lo largo de la vida. Un marco de referencia europeo. Puesta en práctica del programa educación y formación 2010. Recuperado de: http://www.educastur.princast.es/info/calidad/indicadores/doc/ comision_ europea.pdf

Comisión Europea (2007). Proyecto Tuning América Latina. Recuperado de: http://tuning. unideusto.org/tuningal/index.php?d $=0 \&$ option $=$ com_frontpage\&Itemid $=1\langle=e s$

Dirección General de Educación y Cultura. (2002) Informe Eurydice. Las Competencias Clave. Madrid: CIDE.

Key Competencies (2000) A developing concept in general compulsory education. Eurydice

Le Bortef, G. (2001) Ingeniería de las Competencias. Barcelona: Gestión.

López Martínez, J. (2012) Las competencias básicas como lugar de encuentro. Madrid: Conocimiento Educativo.

Pérez Gómez, A. (2008), ¿Competencias o pensamiento práctico? La construcción de los significados de representación y de acción, en J. Gimeno (comp.), Educar en competencias ¿quué hay de nuevo?. Madrid: Morata

Perrenoud, P. (2004). Diez nuevas competencias para enseñar. Barcelona: Graó.

Rychen, D.S. y L.H. Salganik (2006) Las competencias clave para el bienestar personal, social y económico. Málaga: Aljibe.

Weinert, F.E. (1999), Concepts of competence, Munich: Max Planck Institute for Psychological Research.

Fecha de recepción: 10 de marzo2015

Fecha de aceptación: 28 de abril 2015 